\title{
Breastfeeding Practices in Kathmandu
}

\author{
Mathema $\mathbf{S}^{1}$
}

\begin{abstract}
Introduction: In spite of significant evidence on a number of important health benefits of breastfeeding, it has been seen that a vast number of infants in Nepal are not being breastfed appropriately based on standard recommendations. This study was conducted to evaluate the breastfeeding practices prevalent in Kathmandu. Materials and Methods: This was a cross-sectional survey carried out over a period of 24 months from January 2011 to December 2012 on a convenient sample of children aged 6 months to 23 months when they were brought for treatment to Kathmandu Medical College and Teaching Hospital. Breastfeeding was defined according to the recent WHO guidelines and mothers were interviewed as per a structured questionnaire. Results: There were 1250 children enrolled in the study, out of which almost all infants $(99.7 \%, n=1246)$ received colostrum. Only $62.64 \%(n=783)$ babies received breast milk as their first food. Initiation of breastfeeding within one hour of delivery was done by 719 (57.5\%) mothers. The median duration of exclusive breastfeeding was 3.8 months. Conclusion: This study reflects that majority of infants in Kathmandu are still not fed in accordance with breastfeeding recommendations and guidelines. More attention should be given to a proper community-based program including subsequent monitoring of its implementation as exclusive breastfeeding has been proved to be one of the most effective ways to improve infant survival.
\end{abstract}

Key words: Breastfeeding, Infant nutrition, Breast milk, Kathmandu

\section{Introduction}

R reastfeeding, particularly exclusive breastfeeding, for the initial B six months of life, provides better health for both infants and mothers ${ }^{1}$. Breast milk gives infants all the nutrients they need for healthy development. Breast milk is affordable and readily available. It contains antibodies that help protect infants from common childhood illnesses such as diarrhoea and pneumonia, the two primary causes of child mortality worldwide. Breastfed infants have enhanced immune response and reduced risk for chronic illnesses such as asthma, diabetes and inflammatory bowel disease ${ }^{2}$. Breastfeeding also benefits mothers by reducing the risk of breast and ovarian cancer, providing a natural method of birth control and
'Dr. Smriti Mathema, MBBS, MD, Lecturer, Department of Paediatrics, Kathmandu Medical College and Teaching Hospital, Kathmandu, Nepal

\author{
Address for correspondence: \\ Dr. Smriti Mathema, Lecturer \\ Department of Paediatrics \\ Kathmandu Medical College and Teaching \\ Hospital, Sinamangal, Kathmandu, Nepal \\ E-mail: smritimathema@hotmail.com
}

\section{How to cite}

Mathema S. Breastfeeding Practices in Kathmandu. J Nepal Paediatr Soc 2014;34(2):96-99.

doi: http://dx.doi.org/10.3126/jnps.v34i2.10032

This work is licensed under a Creative Commons Attribution 3.0 License.

\section{(c) (i)}

enhancing emotional health of the mother by the bonding she develops with her infant during breastfeeding ${ }^{3,4}$.

Review of evidence has shown that, on a population basis, exclusive breastfeeding for six months is the optimal way of feeding infants and has been recommended by the World Health Organization (WHO $)^{5}$. However, mothers receive infant feeding information and advice from a variety of sources ${ }^{6}$ and it is often found in many cultures that this recommendation of exclusive breastfeeding is not followed by many mothers.

The aim of this study was to evaluate the breastfeeding practices prevalent in Kathmandu. 


\section{Materials and Methods}

A cross-sectional survey was carried out over a period of 24 months from January 2011 to December 2012 on a convenient sample of children aged 6 months to 23 months. Mothers of children belonging to this age group who brought them for treatment to Kathmandu Medical College and Teaching Hospital (KMCTH), Sinamangal, Kathmandu, Nepal were interviewed as per a structured questionnaire.

Breastfeeding was defined according to the recent WHO guidelines ${ }^{7}$ and was categorized as follows:

Exclusive breastfeeding:The infant had received only breast milk from his/her mother or a wet nurse, or expressed breast milk and no other liquids or solids with the exception of drops of syrup consisting of vitamins, mineral supplements or medicines.

Predominant breastfeeding: The infant's predominant source of nourishment had been breast milk. However, the infant may also have received water and water-based drinks like tea and local herbal drops.

Partial breastfeeding: When infant's feeding included non-breast milk foods such as animal/ powdered/condensed milk and/or solid/semi-solid food (i.e. cereals, vegetables, fruits, lentils or meat).

Children with delayed developmental milestones, illness during neonatal period, children with specific feeding problems secondary to congenital malformations and those whose mothers were not accompanying the child during the hospital visit were excluded from the study.

\section{Results}

In this study, a total of 1250 mothers of children between the age group of 6 months to 23 months of age were interviewed when they brought the child for treatment to $\mathrm{KMCTH} .44 \%$ of the mothers interviewed were attending the hospital with their first born child and $56 \%$ had more children.

All the women had at least one antenatal checkup during the last pregnancy, but only $18 \%$ of them reported to have received information on breastfeeding during their antenatal visit. Out of all the women, only seven of them said they had their breasts/nipples examined during the antenatal check-up.
The mothers fell between the age range of 19-38 years. 477 (38.2\%) women were working outside home. The first choice of advice the mother would turn to if her baby had a problem in breastfeeding is shown in Figure 1.

Almost all infants $(99.7 \%, \mathrm{n}=1246)$ received colostrum. Only $62.64 \%(n=783)$ babies received breast milk as their first food. Remaining, 464 $(37.12 \%)$ received dried-milk infant formula, two $(0.16 \%)$ received water and one $(0.08 \%)$ was given glucose water as represented in Figure 2. Initiation of breastfeeding within one hour of delivery was done by 719 (57.5\%) mothers. Additionally, nearly half of all breastfed infants (48.4\%) were supplemented with infant formula during the post-delivery hospital stay. Only 11 mothers $(0.88 \%)$ reported the need to express breast milk, out of which eight mothers expressed manually and three mothers used breast pumps.

The proportion of exclusive breastfed infants at one month, four months and six months is depicted in Figure 3. The median duration of exclusive breastfeeding was 3.8 months. The commonest reason for starting other forms of feeding (infant formula/ animal milk/ semi-solid) was the mother's perception of 'not enough breast milk'. Out of the mothers using dried-milk infant formulae, only $54.6 \%$ were using the proper dilution of formula. $92.7 \%$ of these mothers used bottle feeding and remaining $7.3 \%$ were fed with spoon.

Use of animal milk before the age of six months was found in 427 (34.2\%) infants. All of them except four had used cow's milk.

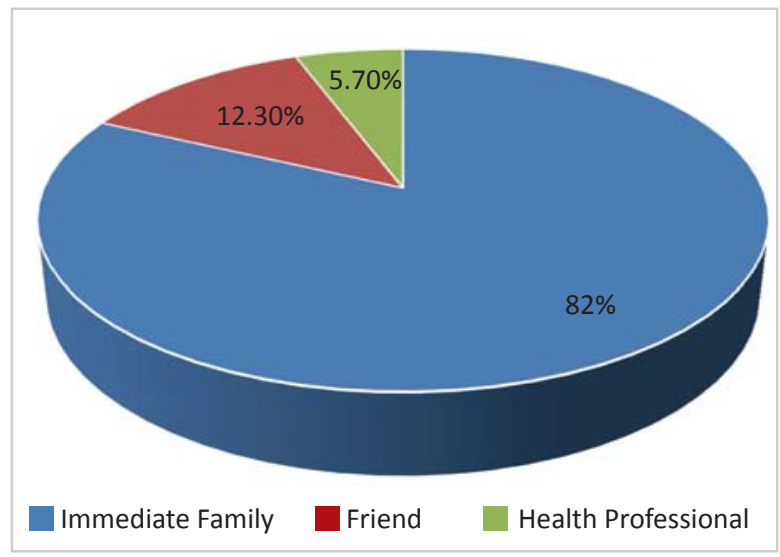

Fig 1: First Choice for Advice the Mother would approach in case of a Breastfeeding Problem 


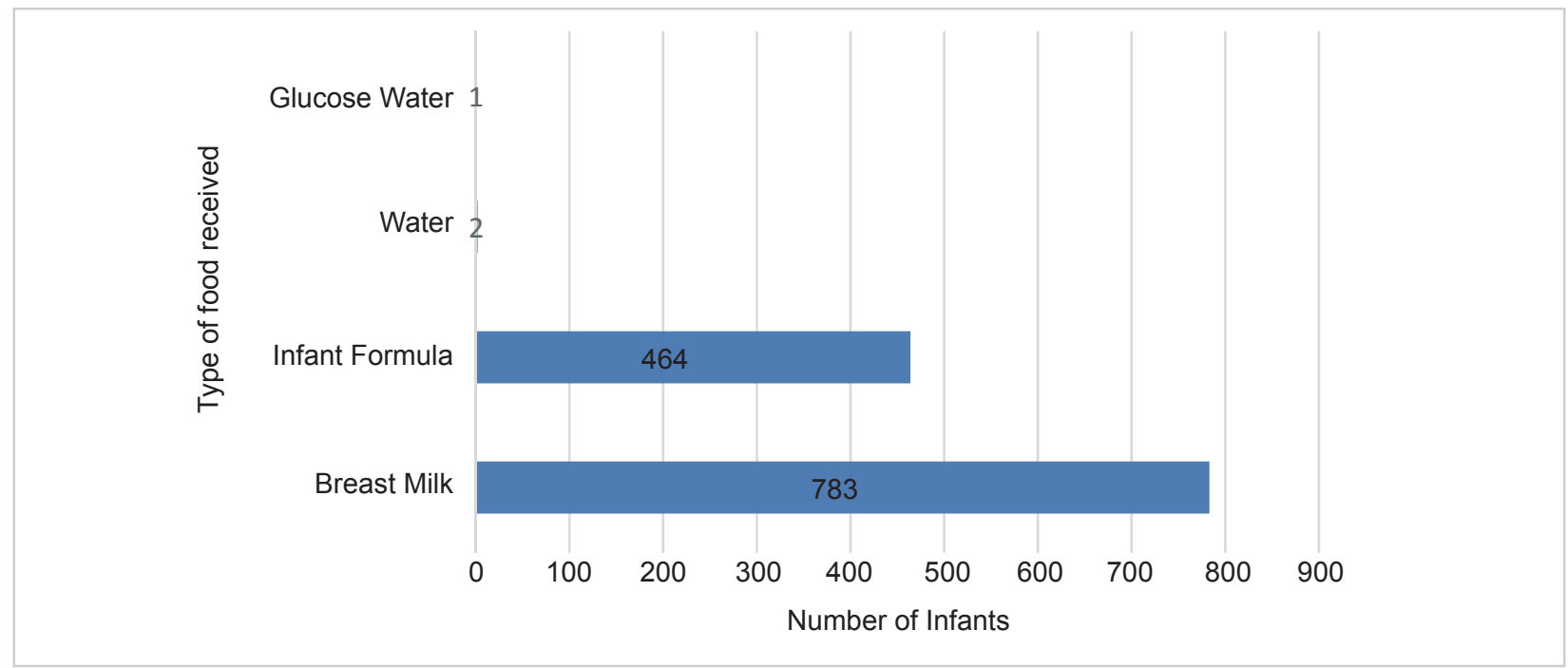

Fig 2: Various Types of First Food received by the Baby immediately after birth

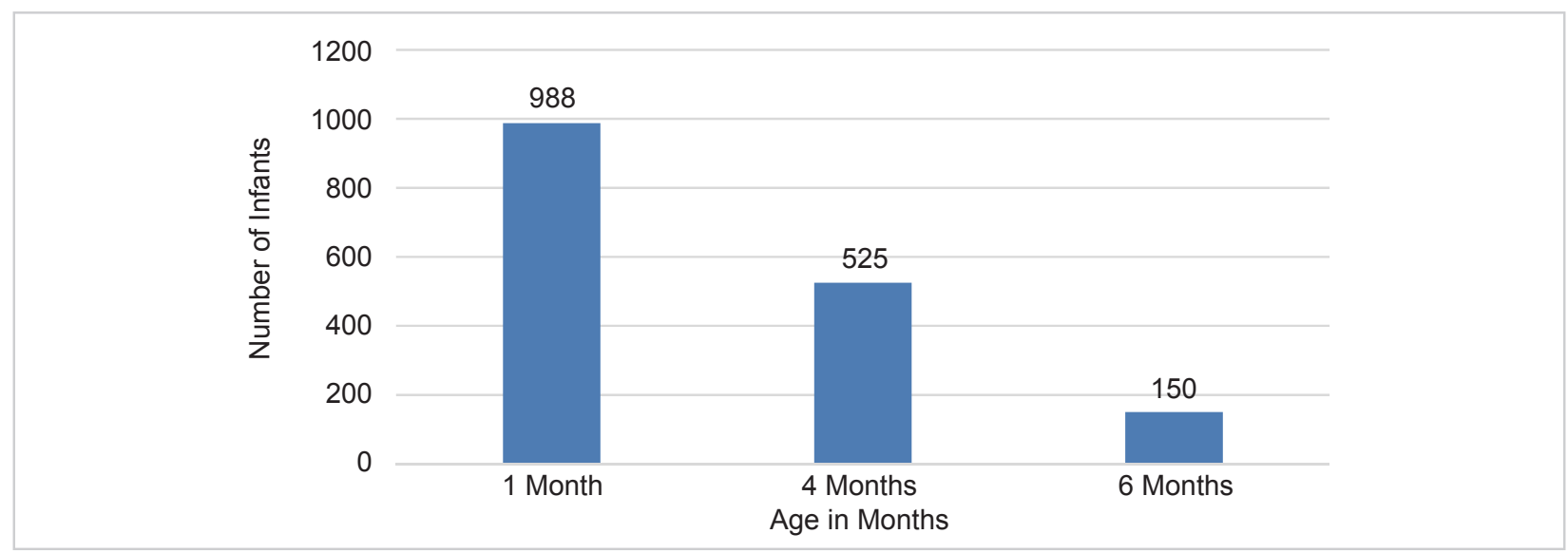

Fig 3: Proportion of Exclusive Breastfed Infants at 1, 4 and 6 months of age

\section{Discussion}

American Academy of Pediatrics (AAP) recommends exclusive breastfeeding for the first six months with continued breastfeeding past 12 months $^{8}$. In spite of these recommendations and proved benefits, it has been seen that only one-fourth of children age 6-23 months in Nepal are fed appropriately based on recommended Infant and Young Child Feeding (IYCF) practices $^{9}$.

National Demographic Health Survey (NDHS) in $2011^{9}$ states that breastfeeding is nearly universal in Nepal, $70 \%$ of children less than age six months are exclusively breastfed, and the median duration of exclusive breastfeeding is 4.2 months. However, this study has found a low prevalence of exclusive breastfeeding at six months of age (12\%) in Kathmandu with a median duration of exclusive breastfeeding being 3.8 months. Similar low prevalence of $9 \%$ has been found in a recent study done in Bhaktapur District by Ulak et $\mathrm{al}^{10}$.

Almost all infants received colostrum but only about two thirds (62.64\%) babies received breast milk as their first food. Some practices, including prelacteal feeding and supplemental feeding, which are not recommended by WHO, are still common in Kathmandu. Widespread use of infant milk formula, even in the hospital set-up, is observed. Infant formula and/or early introduction of semi-solid is done and as found in this study it often occurs when mothers perceive that they have insufficient breast milk production $^{11}$.

Only $18 \%$ women reported to have received information on breastfeeding during their antenatal check-up. This is suggestive of how antenatal visit focuses mainly on pregnancy, and there is not much emphasis on breastfeeding education. 
Although breastfeeding is a social norm and universal practice in most communities, it is also a learned behavior and may be influenced by many factors including socio-economic, educational level and cultural ${ }^{12}$. The encouragement of breastfeeding to families and to the community at large is an important aspect of the paediatrician's profession ${ }^{13}$.

\section{Conclusion}

This study on breastfeeding practices indicate that majority of infants in Kathmandu are not fed in accordance with breastfeeding recommendations and guidelines. The duration of exclusive breastfeeding is shorter than recommended with only about two thirds babies receiving breast milk as their first food. Prelacteal, supplemental feeding and early introduction of semisolid feeds still seem to be common in Kathmandu.

\section{Recommendations}

Important advocacy and advice to encourage breastfeeding is lacking in the society. Although mothers tend to adhere to advice from health professionals, they are not likely to ask for help from them when faced with challenges in doing so. There is also need to overcome clinical barriers to help support initiation of breastfeeding within one hour of birth. Breastfeeding counseling at antenatal clinics and peer support for exclusive breastfeeding should be included as part of breastfeeding promotion programs.

Acknowledgements: None

Funding: Nil

Conflict of Interest: None

Permission from IRB: Yes

\section{References}

1. Stuebe AM: The risks of not breastfeeding for mothers and infants. Rev Obstet Gynecol 2009;2(4):222-231.

2. Oddy WH. Breastfeeding protects against illness and infections in infants and children: A review of the evidence. Breastfeed Rev 2001:9(2):11-18.

3. Labbok M.H., Effects of Breastfeeding on the
Mother, Pediatr Clin North Am 2001:48(1): 143158.

4. Lipworth L, Bailey LR, Trichopoulous D. History of Breast-feeding in Relation to Breast Cancer Risk: A Review of the Epidemiologic Literature. J Natl Cancer Inst 2000:92(4) 302-312.

5. The optimal duration of exclusive breastfeeding: Reports of an expert consultation. Geneva, WHO; 2001.

6. Carruth BR, Skinner JD. Mothers' sources of information about feeding their children ages 2 months to 54 months. J Nutr Educ 2001:33(3):143147.

7. World Health Organization: Indicators for Assessing Infant and Young Child Feeding Practices: Conclusions of a consensus meeting held 6-8 November 2007 in Washington D.C., USA. 2007.

8. Eidelman Al, Schanler RJ, Johnston M, Landers S, Nable L, Szucs K, Viehmann L, Feldman-Winter L, Lawrence R, Kim S, Onyema N. Breastfeeding $\mathrm{S}$ : Breastfeeding and the use of human milk. Pediatrics 2012;129(3):E827-E841.

9. Ministry of Health and Population (MOHP) [Nepal], New ERA, and ICF International Inc. 2012. Nepal Demographic and Health Survey 2011. Kathmandu, Nepal: Ministry of Health and Population, New ERA, and ICF International, Calverton, Maryland.

10. Ulak M, Chandyo RK, Mellander L, Shrestha PS, Strand TA. Infant feeding practices in Bhaktapur, Nepal: a cross-sectional, health facility based survey. Int Breastfeed J 2012;8:1. doi: 10.1186/1746-4358-7-1.

11. Binns CW, Scott JA: Breastfeeding: reasons for starting, reasons for stopping and problems along the way. Breastfeed Rev 2002;10(2):13-19.

12. Aryal TR. Breastfeeding in Nepal: patterns and determinants. J Nepal Med Assoc 2007;46(165):1319.

13. Handa D, RJ Schanler: Role of the Pediatrician in breastfeeding management: Pediatr Clin N Am 2013;60:1-10. 